ESJ Social Sciences

\title{
Revue de Litterature du Concept « Performance Logistique » : Un Essai de Synthese
}

\author{
Alae El Bakkouri, Docteur en Sciences de gestion \\ Université Ibn Zohr, Agadir, Maroc
}

Doi:10.19044/esj.2021.v17n23p210

Submitted: 11 June 2021

Accepted: 05 July 2021

Published: 31 July 2021
Copyright 2021 Author(s)

Under Creative Commons BY-NC-ND

4.0 OPEN ACCESS

Cite As:

El Bakkouri A.(2021). Revue de Litterature du Concept «Performance Logistique » : Un Essai de Synthese. European Scientific Journal, ESJ, 17(23), 210.

https://doi.org/10.19044/esj.2021.v17n23p210

\section{Résumé}

La performance est une notion complexe, ambiguë et difficile à définir. Auparavant, elle se focalise sur les notions d'efficacité et d'efficience (la réduction des coûts) et elle est la plupart du temps centrée sur la dimension financière. En effet, l'aspect multidimensionnel de la performance est aujourd'hui confirmé, et elle devient un concept qui intègre différentes dimensions pour la définir et différents indicateurs de mesure. Dans le contexte de la chaîne logistique, la performance logistique a fait l'objet de nombreux travaux de recherche, notamment ces dernières années. Certaines recherches s'intéressaient à l'amélioration du fonctionnement du processus productif et à l'optimisation de la planification. D'autres recherches ont étudié les facteurs clés de la performance organisationnelle. Ce présent travail aura comme objectif central de présenter la diversité des regards possibles sur la notion de performance logistique et ses différents indicateurs et référentiels.

Mots clés : Performance ; indicateur de la performance, performance logistique ; modèles d'évaluation de la performance logistique 


\title{
Literature Review of « Logistic Performance » Concept : A Synthesis Essay
}

\author{
Alae El Bakkouri, Docteur en Sciences de gestion \\ Université Ibn Zohr, Agadir, Maroc
}

\begin{abstract}
Performance is a complex concept, ambiguous and difficult to define. Previously, it focused on the concepts of effectiveness and efficiency (cost reduction) and it is mostly focused on the financial dimension. Indeed, the multidimensional aspect of performance is now confirmed, and it is becoming a concept that integrates different dimensions to define it and different measurement indicators. In the context of the supply chain, logistics performance has been the subject of much research, especially in recent years. Some research was interested in improving the functioning of the production process and optimizing planning. Other research has looked at the key factors in organizational performance. The main objective of this present work will be to present the diversity of possible views on the notion of logistics performance and its various indicators and benchmarks.
\end{abstract}

Keywords : Performance ; performance indicator, logistics performance ; logistics performance assessment models

\section{Introduction}

Aujourd'hui, toute entreprise est confrontée à de nombreux défis tels que l'augmentation du marché de la concurrence, la mondialisation des marchés, l'évolution technologique, l'évolution des attentes et des besoins... auxquels leur performance est très sensible. En conséquence, le contexte économique actuel du marché pousse les entreprises à rationaliser au maximum leurs modes de gestion, et adopter des nouvelles formes d'organisation.

La mesure de la performance est l'une des problématiques présentes de manière récurrente dans de nombreuses recherches académiques, particulièrement dans un contexte de chaîne logistique (Sovorons et Zipkin, 1988 ; Tan, 2001 ; Min et Zhou, 2002 ; Arzu et al., 2010 ; Yao et al., 2006 ; Kabili et al., 2010), où l'on traite à la fois de la performance individuelle de chacun des acteurs, et de la performance globale de toute la chaîne.

La performance logistique attire beaucoup l'attention des chercheurs, ainsi que celle des managers, car elle reflète une partie importante de la performance globale de l'entreprise (Lehtonen, 2001). De plus, la performance globale n'est pas la simple somme des performances individuelles, 
conformément aux principes de la systémique (Botta-Genoulaz et al. 2010). C'est pourquoi, la mesure de la performance n'est pas un objectif en soi mais un moyen mis à la disposition des entreprises pour leur progression.

L'objectif central de ce travail, est de présenter la diversité des regards possibles sur la notion de performance logistique ainsi que ses différents indicateurs et référentiels.

Pour ce faire, et afin de mieux cerner la notion de performance logistique, il s'avère nécessaire d'exposer le concept de performance dans sa globalité et ses différentes approches. Par la suite, nous entamons l'examen et l'analyse des travaux sur la performance logistique. A la fin, nous étudions les différents indicateurs et modèles d'évaluation de la performance logistique.

\section{La performance : cadre conceptuel \\ 1.1. Définitions}

Associé à tout système ou activité, le terme performance est largement utilisé et étudié dans son concept, sa mesure et son mode de gestion. Afin de mieux cerner la notion de performance logistique, il s'avère tout d'abord nécessaire d'exposer le concept de performance dans sa globalité.

Par nature, la notion de la performance est multidimensionnelle (Chow et al., 1994) surtout lorsqu'on s'intéresse aux structures interorganisationnelles où le nombre de partenaire reste important, et sa définition a toujours été une préoccupation majeure des entreprises (Estampe et al., 2013).

Dans le domaine de la gestion des entreprises, la performance a toujours été une notion ambiguë et rarement définie explicitement (Issor, 2017). C'est dans ce sens que Lebas et Euske (2007) déclarent que le mot « Performance » est amplement utilisé dans tous les domaines de la gestion. " On trouve dans le domaine du contrôle de gestion, des termes tels que la gestion de la performance, l'évaluation de la performance et l'estimation de la performance ». En effet, le concept de la performance reste une problématique largement abordée dans la littérature grâce à ses éclairages qu'elle offre aux gestionnaires dans la gestion stratégique de leurs organisations (Giraud et al., 2002). Ce concept de performance est couramment utilisé dans les milieux organisationnels pour désigner un certain niveau d'excellence.

La performance est qualifiée selon, les gestionnaires comme étant « tout ce qui, et seulement ce qui, contribue à atteindre les objectifs stratégiques $»$ (Lorino, 1997). En outre, la performance des organisations dépend de leurs caractéristiques internes mais également des liens qu'elles nouent avec d'autres organisations. Elle peut être mesurée selon, Chen et Paulrai (2004) de plusieurs façons et de plusieurs points de vue (financière et opérationnelle). De la même manière il n'existe donc pas une seule performance, mais plutôt plusieurs performances (Bronet, 2006). 
Le concept de la performance a connu, depuis son adoption par les gestionnaires, plusieurs significations. Au début, dans les années quatre-vingt la performance d'une entreprise se situait au centre d'un triptyque (figure 1) : objectifs, moyens et résultats (Gibert, 1980).

Figure 1 : Triangle de la performance

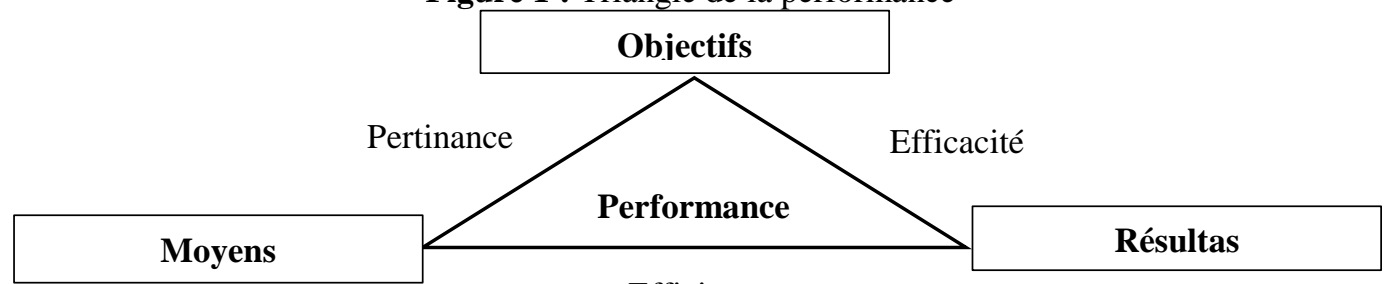

Efficience

Source : Gibert (1980)

Le segment entre résultats et moyens défini l'efficience et permet de savoir si l'entreprise arrive à atteindre ses objectifs avec moins de coûts et de moyens. Le segment entre moyens et objectifs désigne la pertinence et permet de savoir si l'entreprise s'est munie des bons moyens pour atteindre ses objectifs. Le segment entre objectifs et résultat désigne l'efficacité et permet de savoir si l'entreprise a obtenu le résultat souhaité ou attendu. A partir des années 1990 , le périmètre de la performance a étendu pour arriver à la dimension organisationnelle des organisations. Elle désigne la mesure de l'atteinte des objectifs, mais aussi, «mesure de ce qui contribue à atteindre ces objectifs » (Morin et al, 1994). À ce stade, la performance de l'entreprise dépasse l'aspect financier.

Dans la littérature, l'étude de la notion de la performance a occupé une place centrale dans de nombreux recherche. En effet, et pour répondre à la question «Qu'est-ce que la performance ?» nous passons en revue les différentes définitions de la performance proposées dans la littérature. Nous illustrons dans le tableau ci-dessous quelques-unes.

Tableau 1 : Définitions de la performance

\begin{tabular}{|c|l|}
\hline Auteur & \multicolumn{1}{c|}{ Définition } \\
\hline Albanes,1978 & $\begin{array}{l}\text { "La performance de l'entreprise peut se définir comme le degré de "La } \\
\text { performance est la raison des postes de gestion, elle implique l'efficience } \\
\text { et l'efficacité ». }\end{array}$ \\
\hline Berrah, 2015 & $\begin{array}{l}\text { "Une entreprise performante est une entreprise qui atteint les objectifs } \\
\text { qu'elle annonce ». }\end{array}$ \\
\hline Pichot, 2006 & $\begin{array}{l}\text { "La performance est l'efficience et l'efficacité d'une organisation à } \\
\text { réaliser ses objectifs ». }\end{array}$ \\
\hline Notat, 2007 & $\begin{array}{l}\text { "Un constat officiel enregistrant un résultat accompli à un instant t, } \\
\text { toujours en référence à un contexte, à un objectif et un résultat attendu, et } \\
\text { ce quel que soit le domaine ». }\end{array}$ \\
\hline $\begin{array}{c}\text { Armstrong, } \\
2009\end{array}$ & $\begin{array}{l}\text { "C'est un processus systématique qui vise à améliorer la performance } \\
\text { organisationnelle en développant la performance des individus et des } \\
\text { équipes ». }\end{array}$ \\
\hline
\end{tabular}


\begin{tabular}{c|c}
$\begin{array}{c}\text { Wissler et al., } \\
2012\end{array}$ & «Le résultat d'une action ».
\end{tabular}

Source : Traduit et adopté de la littérature

Quatre points communs ont été identifiés à partir des définitions mentionnées ci-dessus :

- Le terme performance désigne un résultat qui représente le «niveau de réalisation des objectifs » (Burlaud, 1995)

- Le terme de performance est souvent utilisé dans un contexte d'évaluation, c'est pour cela dans la performance est intimement liée à la valeur.

- Le terme de performance ne se définit pas toujours d'une manière objective et absolue mais plutôt d'une manière subjective.

- Le terme de performance c'est une action, qui implique une production réelle, donc un processus ;

Il en résulte que la performance peut être vue comme la capacité d'une entreprise à conduire une action pour obtenir des résultats conformément à des objectifs fixés préalablement, tout en minimisant le coût des ressources et des processus mis en œuvre (Neely et Platts 2005).

De ce qui précède, la performance a pour objectif de maximiser la création de la valeur, et de minimiser les coûts de l'entité en question. De plus, elle est un concept qui s'attache à un résultat obtenu, lié à l'atteinte d'un objectif et, de manière équivalente, à l'exécution d'une action. En somme et comme démontré là-dessus, la notion de performance est reconnue par plusieurs auteurs par son caractère multidimensionnel et elle est intéressante pour les entreprises de la mesurer à différents niveaux.

\subsection{Les indicateurs de la performance}

Un critère est une valeur définie qui sert de base à un jugement et qui permet de mesurer la performance. Les critères de performance constituent des repères et permettent aux entreprises de mener une action en vue d'atteindre un objectif. Alors, les indicateurs de performance d'une entreprise sont à la fois un outil de mesure de la santé de l'entreprise et un outil d'aide à la décision.

Les critères peuvent être d'ordre quantitatifs ou qualitatifs. Ils sont définis en fonction des objectifs propres à chaque organisation. En économie, les indicateurs sont des statistiques construites afin de mesurer certaines dimensions de l'activité économique et leurs évolutions. Alors, ils se présentent sous forme des informations concrètes, chiffrées et opérationnelles qui fournissent des indications, des renseignements sur la valeur d'une grandeur mesurée (Larousse, 2012). 
Un indicateur de performance est « une donnée quantifiée qui mesure l'efficacité et/ou l'efficience de tout ou partie d'un processus ou d'un système (réel ou simulé), par rapport à une norme, un plan ou un objectif, déterminé et accepté dans le cadre d'une stratégie d'entreprise » (AFGI, 1992). En fait, les indicateurs de performance ont deux fonctions : premièrement évaluer et mesurer les résultats ou les données déjà réalisés, deuxièmement donner l'information aux décideurs ou aux utilisateurs potentiels pour fixer des objectifs à atteindre dans le futur (Bhagwat et Sharma, 2007). Depuis longtemps, les entreprises ont utilisé les indicateurs pour évaluer leur performance, notamment à travers leur comptabilité.

Les indicateurs de performance sont des données permettant aux décideurs d'entreprises à caractériser une situation évolutive, une action ou les conséquences d'une action de façon à les évaluer et à comparer leur état à différentes dates (NOYES D et al, 2007).

Globalement la performance d'une organisation est évaluée par des indicateurs dites stratégiques. Ceux-ci sont liés à des indicateurs de processus qui traduisent la performance locale de différents services de l'entreprise. Ces indicateurs de processus sont associés aux Facteurs de Performance lorsqu'ils se situent au niveau tactique et aux Facteurs de Progrès pour le niveau opérationnel (Clivillé, 2004).

Pour évaluer la qualité et la pertinence des indicateurs de performance, Lorino (2001) les classent selon trois critères :

- Le critère stratégique de l'indicateur : il doit être associé à un objectif clair à atteindre. Il informe du bon déroulement ou non d'une action qui participe à l'atteinte des objectifs.

- Le critère tactique de l'indicateur : il doit permettre d'orienter facilement l'acteur à agir et comprendre les facteurs de réussite ou d'échec.

- Le critère opérationnel de l'indicateur : il consiste à vérifier que les mesures effectuées soient les résultats d'un type d'action précis et identifié, que les données utilisées soient dignes de confiance. La pertinence opérationnelle d'un indicateur concerne donc la validité des résultats.

Le choix d'un bon critère de mesure de la performance repose selon Dorey F (1986) sur trois conditions

- L'indicateur de performance choisie doit être parfaitement en harmonie avec les phénomènes étudiés.

- L'indicateur de performance choisie doit être objective et mesurable, cela veut dire il doit être calculé à partir des données fiables et disponibles. 
- L'indicateur de performance choisi doit prendre en considération l'aspect dynamique des phénomènes observés.

En somme, un indicateur de performance est un outil facilitant la prise de décision pour les décideurs et qu'il est pour objectif de mesurer la réalisation d'un objectif en temps réel et d'apprécier le plus objectivement possible l'atteinte d'une performance. En effet, le paragraphe suivant a pour mission de présenter un état de l'art de la performance logistique.

\section{La performance logistique : cadre conceptuel}

\subsection{Définitions}

La logistique est maintenant au cœur des priorités de l'entreprise parce qu'elle contribue considérablement à la performance de celle-ci (Pimor, 2005). De plus, le succès d'une entreprise est fortement lié à la performance de sa chaîne logistique. Aujourd'hui, atteindre le niveau de la performance ne peut être obtenu, si on se focalisant seulement sur l'optimisation des processus internes mais nécessite une vision globale pour améliorer la performance de toute la chaîne logistique.

Une logistique est dite performante, si et seulement si l'entreprise est capable d'assurer la satisfaction du client en lui livrant des produits/ services de bonne qualité, en quantité requises, au bon moment, au bon endroit et en consommant moins de ressources. Ainsi, et pour y arriver, il est indispensable de maîtriser toutes les fonctions opérationnelles existantes entre les différents acteurs de la chaîne logistique de l'approvisionnement à la livraison en passant par la production, l'acheminement, l'entreposage et le conditionnement.

Pour atteindre la performance logistique, les entreprises veillent à étudier le rapport entre la qualité de service fourni au client (efficacité) et les moyens consommés à cette intention (efficience) (Richey et al., 2010). Ainsi, la performance logistique est généralement liée au service de livraison, aux coûts logistiques et au coût d'immobilisation du capital (Forslund, 2012). Alors que, le service de livraison se mesure par les délais de livraison, les coûts logistiques sont liés au transport et à l'entreposage, tandis que le coût d'immobilisation du capital concerne les stocks de matières, composants et produits finis.

De façon générale, l'efficacité correspond au respect des exigences de la clientèle, et l'efficience correspond aux ressources de l'organisation utilisées pour atteindre les niveaux escomptés de satisfaction de la clientèle. Ainsi que, une logistique performante est tout élément contribue à optimiser le rapport valeur/ coût dont l'objectif est de minimiser le total des coûts logistiques comme le délai, la flexibilité et le temps de livraison, etc., pour un niveau de service souhaité productivité. 
Bref, La performance logistique est pour mission « de répondre à la demande du client à un niveau de service fixé au moindre coût » (Heskett, 1977). De même, La performance logistique est un concept multiple qui doit être appréhendé de façon globale et transversale dans la mesure où les flux ne s'arrêtent pas aux frontières de l'entreprise.

\subsection{Les critères de la performance logistique}

Dans la chaîne logistique, les flux physiques, d'informations et monétaires ne s'arrêtent pas aux frontières de la même entreprise, mais ils dépassent ces frontières aussi bien en amont qu'en aval, puisqu'on parle du fournisseur du fournisseur au client du client. Face à cette réalité, le concept de la performance logistique est devenu complexe et à multiples facettes et qui doit être appréhendée de façon transversale et globale sur toute la chaîne logistique.

Selon Lorino (2003) et Bouquin (2004), les indicateurs de performance sont associés à une « action à piloter », où ils se résument à faire «mieux que les autres, plus vite, moins cher, plus écologique », en mettant l'accent sur quatre leviers clés, soient la fiabilité, l'efficience, la réactivité et le respect de l'environnement.

- La fiabilité logistique est «la capacité de répondre à la demande du client selon un niveau de service fixé » (Jouenne, 2011). Les indicateurs susceptibles de mesurer la fiabilité logistique sont le taux de service fournisseurs, le taux de service client, taux de fiabilité des prévisions, le taux de service transport, le taux de réclamations, le taux d'absentéisme du personnel, etc.

- L'efficience logistique est le rapport entre efficacité et coût. Il vise à atteindre un objectif avec le minimum de moyens engagés. Les indicateurs susceptibles de mesurer cette efficience logistique sont coût de possession de stock, coût total d'achat, coût de fabrication, coût de transport, etc.

- La réactivité logistique est la capacité de s'adapter rapidement aux fluctuations de la demande. Les indicateurs susceptibles de mesurer cette réactivité logistique : rotation des stocks, time-to-market, vitesse d'écoulement des produits, ratio de tension des flux, cycle order-tocash, cycle cash-to-cash, temps de cycle, de transit, d'attente, etc.

- Le volet de respect de l'environnement, à travers l'adoption de pratiques "vertes" tels que le transport multimodal, mutualisation des infrastructures et du transport, logistique inverse ainsi que le recyclage des produits. Pour les indicateurs éco-logistiques, le pourcentage de la consommation d'énergie, l'émission de gaz à effet de serre les tonnes de CO2 émises par les activités logistiques, etc. 
Dans le même ordre d'idée, la mesure de la performance logistique d'une entreprise, est un élément essentiel, qui fournit aux décideurs d'entreprises en question plus de visibilité autours :

- des informations relatives aux coûts des opérations et des produits qui circulent tout au long de la chaine logistique ;

- des informations nécessaires à l'ensemble des ressources engagées (ressources humaines, financières, technologiques) dans chaque opération d'un processus logistique ;

- des informations relatives aux retours sur investissement réalisé ou dégagés par chaque produit ou chaque opération de ce processus logistique.

Les chercheurs en logistique ont essayé de rassembler les KPI susceptibles de mesurer la performance logistique d'une entreprise. En effet, chaque KPI est appliqué à chaque maillon ou à chaque fonction d'une chaîne logistique, comme le montre le tableau ci-dessous :

Tableau 2 : Exemple d'indicateurs de performance logistique

\begin{tabular}{|c|c|}
\hline Fonction dans l'entreprise & Exemple d'indicateur de performance \\
\hline $\begin{array}{l}\text { La fonction Achat et } \\
\text { Approvisionnement }\end{array}$ & $\begin{array}{l}\text { Taux d'achat par famille de produits, taux de retards, de } \\
\text { litige, etc. }\end{array}$ \\
\hline La fonction Production & $\begin{array}{l}\text { La capacité de production utilisée, le coût d'arrêt des } \\
\text { équipements, la durée moyenne du cycle de production, } \\
\text { etc. }\end{array}$ \\
\hline La fonction Transport & $\begin{array}{c}\text { Taux de remplissage des véhicules, Taux de } \\
\text { consommation du carburant, Traçabilité des véhicules, } \\
\text { etc. }\end{array}$ \\
\hline $\begin{array}{l}\text { La fonction Stockage et } \\
\text { Gestion des stocks }\end{array}$ & $\begin{array}{l}\text { Taux de rotation des stocks, Taux de détention des } \\
\text { stocks, Taux de rupture des stocks, etc. }\end{array}$ \\
\hline $\begin{array}{l}\text { La fonction Vente et } \\
\text { Distribution }\end{array}$ & $\begin{array}{l}\text { Taux de fiabilité des prévisions de ventes, Taux de } \\
\text { satisfaction des clients, etc. }\end{array}$ \\
\hline Le service fourni au Client & Taux du service client, Taux de réclamations client, etc. \\
\hline
\end{tabular}
Source : Nous-même

Pour évaluer le fonctionnement d'une chaine logistique, trois dimensions sont, à notre sens, essentielles à prendre en compte : la vitesse, la qualité et le coût (Hult et al., 2004). C'est ainsi que la performance logistique peut être mesurer par la fiabilité des livraisons, la réduction des coûts, des délais, la conformité aux spécifications des clients, l'amélioration des processus et le time to market ${ }^{1}$.

Les managers des entreprises tentent d'évaluer en permanence leur performance logistique, en utilisant plusieurs indicateurs mis en place par les auteurs du SCM, comme celui de Fassio (2006, p20), qui suppose que la performance logistique est :

\footnotetext{
${ }^{1}$ Time to market désigne le délai de mise sur le marché de nouveaux produits.
} 
- Le respect de la date contractuelle de la livraison au client.

- La livraison au lieu désigné par le client

- La livraison de la juste quantité commandée

- Le respect de la qualité produit prescrite

- La conformité du conditionnement sollicité

- Un ordre de préparation conforme à l'ordre prescrit de la consommation

- L'accompagnement concomitant des informations requises par le client ou la profession.

Ce présent article propose une synthèse des indicateurs tirés depuis les travaux de (Gunasekaran et al., 2004 ; Gunasekaran et al., 2001 ; Barut et al., 2002 ; Sahin et Robinson, 2005 ; Wu et Song, 2005 ; De Toni et Nassimbeni, 2001) pour mesurer la performance d'une chaîne logistique (Figure 2).

Comme indiqué précédemment, trois niveaux distingués des indicateurs de la performance de la chaine logistique (stratégique, tactique et opérationnel), et chaque niveau est rattachés à un macro-processus en différentiant ainsi les indicateurs internes des indicateurs externes. Au niveau stratégique, les indicateurs couvrent les différents processus de la chaîne logistique, ils sont pour la plupart transversaux. En revanche, au niveau tactique et opérationnel, chaque indicateur est associé à un processus.

Figure 2 : Principaux indicateurs de performance d'un supply chain

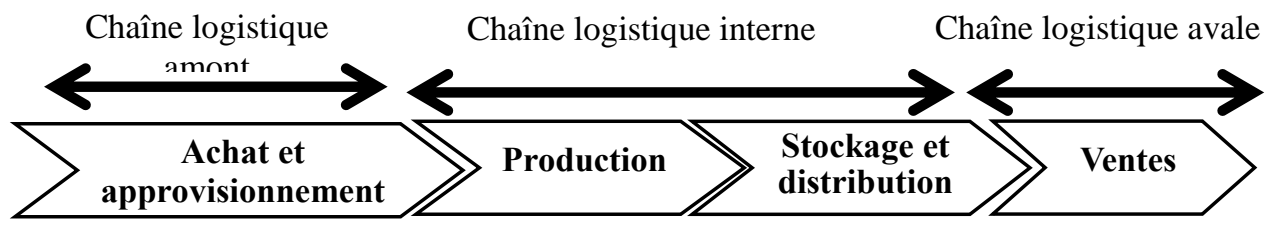

- Indicateurs stratégiques : coût d'achat, profits, délais de la commande etc....

- Indicateurs tactiques : délais fournisseurs, délais de production, fiabilité de la livraison fournisseur, précision des prévisions de ventes etc...

- Indicateurs opérationnels : taux de rebus fournisseurs, taux d'utilisation des ressources, qualité de la livraison etc...

Source : Bonnefous (2001)

Toutefois, et afin d'arriver à mesurer efficacement la performance logistique des entreprises, il fallait demander quels outils ou quels modèles à mettre en place. C'est dans ce sens que plusieurs modèles de mesure de la 
performance logistique ont été élaborés par les auteurs de la SCM. Le paragraphe suivant essaye de mettre en lumière ces différents modèles

\section{Les modèles d'évaluation de la performance logistique}

Les modèles d'évaluation de la performance sont considérés comme des outils de gestion permettant les changements stratégiques. Une de leurs caractéristiques est de représenter plusieurs points de vue (financiers, stratégie, opération, etc.) concernant l'organisation de la chaîne logistique pour permettre une analyse dans le temps et l'espace (Estampe, 2015). En effet, un modèle d'évaluation de la performance adapté est un modèle en cohérence avec les objectifs globaux de l'entreprise (Berrah, 1997 ; Le Clainche, 2001). Il doit permettre de structurer et de lier ces différents indicateurs entre eux, mais il doit également les mettre en perspective, afin de faciliter l'analyse et le diagnostic de sa situation ou de son contexte.

Dans la littérature scientifique, les modèles d'évaluation de la performance logistique ne sont pas nombreux, l'étude des références bibliographiques, ainsi que l'analyse des pratiques de certaines organismes $\left(\mathrm{SCC}^{2}, \mathrm{ASLOG}^{3}\right)$ et personnes (Gilles Paché, Dominique Estampe, Tomas Hult et Angappa Gunasekaran) éminentes dans le domaine de la chaîne logistique, nous a permis d'identifier différents référentiels d'évaluation de la performance logistique. De plus, vu la modernisation, la digitalisation et la mondialisation du concept et des pratiques liés à la chaîne logique, quatre référentiels majeurs méritent d'être cités dans ce présent article dont les suivants :

\section{a- Le modèle WCL (World Class Logistic model)}

Le WCL est un outil regroupant les facteurs-clés de succès d'une politique de chaîne logistique en fonction de quatre domaines de compétences : le positionnement stratégique, l'intégration, la réactivité et la mesure de la performance. Ces quatre domaines fournissent un certain nombre d'indicateurs multidimensionnels de performance pour une meilleure coordination inter organisationnelle, entendue comme l'ensemble des liaisons verticales existant entre les différents intervenants de la chaîne globale qui va $\mathrm{du}$ fournisseur au client final, en passant par les industries de transformation, le canal de distribution et les prestataires de services logistiques (Paché et Sauvage, 1999). Dans le World Class Logistic model, l'un des quatre domaines de compétences considérés correspond à l'intégration de la chaîne

${ }^{2}$ Le Supply Chain Council est une organisation sans but lucratif regroupant plus de 1000 membres, qui sont des professionnels provenant de tous les types d'industries (fabricants, distributeurs, détaillants, services etc.

${ }^{3}$ ASsociation de la LOgistique fédère plus de 500 entreprises et 4000 professionnels. Elle traite toutes les questions relatives aux enjeux de la chaîne d'approvisionnement. 
logistique. L'ensemble des questions relatives à cet axe permet d'évaluer les moyens mis en place pour synchroniser l'ensemble des entités de la supply chain. Ces questions se subdivisent en différents thèmes comme le montre le tableau 3. Le modèle WCL permet également d'évaluer la performance de la supply chain en étudiant plus particulièrement les indicateurs internes de l'entreprise, la manière dont ces indicateurs sont utilisés le long de la chaîne logistique et en proposant une analyse de type benchmark. En revanche, le modèle ne propose pas de liens entre les résultats de l'axe « intégration » et les résultats de l'axe «mesure de la performance ».

Tableau 3 : Une partie du modèle World Class Logistic

\begin{tabular}{|c|c|}
\hline Indicateur & Signification \\
\hline $\begin{array}{l}\text { Unification de la } \\
\text { supply chain }\end{array}$ & $\begin{array}{l}\text { Capacité à développer des relations de coopérations avec les autres } \\
\text { entreprises à travers la chaîne logistique }\end{array}$ \\
\hline $\begin{array}{c}\text { Système } \\
\text { d'information }\end{array}$ & $\begin{array}{l}\text { Investissements en matériels, logiciels et réseaux ainsi que leur } \\
\text { adaptation pour faciliter les processus et les échanges } \\
\text { d'informations sur la chaîne logistique. }\end{array}$ \\
\hline $\begin{array}{l}\text { Partage de } \\
\text { l'information }\end{array}$ & $\begin{array}{l}\text { Volonté d'échanger des données essentielles à caractère technique, } \\
\text { financier, opérationnel et stratégique. }\end{array}$ \\
\hline Compatibilité & $\begin{array}{l}\text { Capacité de l'entreprise à échanger des informations dans un } \\
\text { format approprié, réactif, et facilement utilisable sur la chaîne } \\
\text { logistique }\end{array}$ \\
\hline Standardisation & $\begin{array}{l}\text { Mise en place de politiques et de procédures communes pour } \\
\text { faciliter et améliorer les opérations logistiques }\end{array}$ \\
\hline Simplification & Re-engineering des procédures afin d'améliorer l'efficacité \\
\hline $\begin{array}{c}\text { Adhésion des } \\
\text { hommes }\end{array}$ & Acceptation des politiques et des procédures opérationnelles. \\
\hline
\end{tabular}

Source : Paché et Sauvage (1999)

\section{b- Le référentiel SCOR (Supply Chain Operations Reference)}

Initialisé en 1996 par le Supply Chain Council (S.C.C), ce modèle présente un guide standard pour les entreprises, qui définit une démarche, des processus, des indicateurs et les meilleures pratiques du moment pour représenter, évaluer et diagnostiquer la chaîne logistique. Ce modèle replace la chaîne logistique interne de l'entreprise au sein de la chaîne logistique étendue avec clients et fournisseurs. Il identifie cinq processus de niveau 1 (voir Figure 3) :

- Processus «Planifier » qui correspond à la mise en adéquation des capacités avec la demande (planification des stocks, priorisation des demandes, ...).

- Processus « Approvisionner » qui regroupe l'ensemble des actions concourant à la mise à disposition des matières premières ou services conformément aux plannings incluant notamment la commande, la réception, le contrôle des matières. 
- Processus «Fabriquer » qui contient l'ensemble des actions de transformation du produit.

- Processus « Distribuer » qui correspond à l'ensemble des étapes qui permettent de livrer le produit conformément à la demande (gestion de la commande, stocks, transport...).

- Enfin, Processus « Retour » qui a été intégré au modèle de référence à partir de la version 4.0 (SCC. 2000) et qui ne cesse d'évoluer depuis et qui regroupe les activités de pilotage des flux retours des produits incluant entre autres, les fonctions de planification, de réception et de vérification.

Figure 3 : Niveau 1 modèle SCOR

Source : Supply Chain Council (2005)

Un processus supplémentaire est introduit par le modèle SCOR : il s'agit du processus «Enable». Il représente les activités supports à la gestion de la chaîne logistique, les différentes tâches et informations utiles pour la réalisation des processus " opérationnels ». Ce processus est scindé en plusieurs sous processus «Enable » planifier, approvisionner, fabriquer, distribuer et retourner. Chacun de ces processus est ensuite décrit sur 3 niveaux supplémentaires. Le niveau 2, les catégories de processus, correspond à une déclinaison des processus de niveau 1 en fonction de la stratégie logistique. Il permet de préciser les processus et de simplifier leur compréhension.

\section{c- Le guide logistique ASLOG}

Le guide logistique ASLOG est un référentiel logistique en se basant sur celui mis au point par Volvo dans les années 1990. Ce dernier a été amélioré et constitue à ce jour une base de référence intéressante pour juger de la pertinence d'un système logistique. Le référentiel de la performance logistique de l'ASLOG (ASLOG 2006) est un catalogue de mesures et d'actions de progrès. Le concept de la chaîne logistique a été introduit en 2002, avec la 3ème version, celle-ci étant encore enrichie dans sa version 2005. En effet, Ce référentiel permet de caractériser la situation actuelle de la chaîne 
logistique ainsi que l'évaluation de sa performance à travers 200 questions selon dix axes

- Axe1 : le management, la stratégie, et la planification. Le référentiel préconise un choix de lieu de production en fonction de l'environnement général, des infrastructures, de la réglementation, des possibilités offertes par les collectivités locales, du bassin d'emploi...

- Axe 2 : la conception des produits. Le référentiel encourage la standardisation et la création de modules, la réduction des impacts environnementaux liés à la destruction des emballages,

- Axe 3 : les approvisionnements. Le référentiel recommande de choisir les fournisseurs en fonction de l'éloignement, de la facilité et des coûts de transport ; d'optimiser le transport en partenariat avec d'autres fournisseurs,

- Axe 4 : la production. Le référentiel encourage notamment la polyvalence du personnel et l'organisation de la maintenance préventive afin de limiter les risques de pannes,

- Axe 5 : les livraisons. Le référentiel distingue les livraisons amont et aval mais rien n'est spécifié sur les aspects environnementaux et sociaux,

- Axe 6: le stockage. Une gestion préventive des moyens de manutention et de stockage, la réduction du nombre de références (types, variétés) et la standardisation des conditionnements sont recherchées,

- Axe 7 : les ventes. La fiabilité des informations notées sur l'étiquetage, l'interrogation des clients sur leur satisfaction, ... sont encouragées,

- Axe $8:$ les retours et le SAV. Le référentiel incite à mettre en place des stratégies d'améliorations continues afin de réduire le nombre de retours,

- Axe 9: les indicateurs de pilotage. Le référentiel approuve la mise en place d'un ensemble d'indicateurs synthétiques comme détaillés, permanents comme temporaires,

- Axe 10 : et le progrès permanent. Le référentiel conseille la certification aux normes ISO environnementales. Ces performances sont mesurées grâce à une échelle de cotation allant de 0 à $3(0$ : en l'absence du minimum nécessaire à l'obtention du niveau $1 ; 1,2$ et 3 ; 3 étant le meilleur niveau).

A partir de ces éléments de caractérisation, les auditeurs de l'ASLOG analysent la situation de l'entreprise au sein de sa chaîne logistique et formulent un certain nombre de recommandations permettant une amélioration à venir. 


\section{d- Le référentiel EVALOG}

Le référentiel EVALOG est proposé par un Groupement des industriel appelé « Groupement pour l'amélioration des liaisons dans l'industrie automobile » GALIA. Ce modèle a été développé par les constructeurs et les équipementiers de l'automobile. C'est un guide d'évaluation commun aux fournisseurs et aux clients du secteur, mais peut être utilisé dans d'autres industries. Son objectif consiste à identifier les domaines où les entreprises ont à s'améliorer pour fiabiliser leurs flux physiques et logistiques. Il axe son analyse sur six thématiques dont quatre sont de type processus : relation client, relation fournisseur, production et développement du produit et deux sont des axes d'étude concernant la stratégie de l'entreprise et son organisation. Il s'agit d'évaluer de façon binaire ( 0 pas mise en place, 1 mise en place) une soixantaine de pratiques. La dernière version du référentiel propose quelques recommandations liées à la responsabilité sociale s'inscrivant dans six axes :

- Stratégie et amélioration : le référentiel recommande d'intégrer le processus logistique dans la stratégie de l'entreprise en y incorporant objectifs, analyses et plans d'actions.

- Organisation du travail : le référentiel préconise la maîtrise et l'amélioration des conditions de travail.

- Capacité et planning de production : cet axe permet de comparer les ressources d'une entreprise avec les besoins de ses clients et notamment le lien avec le développement des produits.

- Interfaces clients : Sont évoquées la gestion des emballages, notamment la quantité et le contrôle de celle-ci afin d'éviter les gaspillages ; de même que la politique de transport qui doit participer à la sauvegarde de l'environnement.

- Maîtrise des produits/processus : sont invoqués dans cet axe, la gestion de la traçabilité, la réduction des produits en fin de vie et la gestion des produits défectueux/obsolètes.

- Interface fournisseurs : dans ce processus les problématiques d'emballage sont abordés, le modèle recommande « des emballages appropriés garantissant [...] le respect de l'environnement » et les problématiques de transport qui doivent être «basées sur les problèmes d'environnement ».

Estampe et al. (2013) ont analysé les différences entre ces modèles et ont proposé une grille pour aider le manager à choisir le modèle le plus approprié à ses besoins pour évaluer sa performance. Ces différents modèles cherchent à évaluer la performance de la chaîne logistique d'une organisation et non pas la performance de l'ensemble de la SC des différents maillons de cette chaîne. Toutefois, Gunasekaran et al. (2004) et Hult et al. (2004) 
proposent des listes d'indicateurs utiles pour évaluer la performance de la chaîne logistique dont l'objectif est de proposer aux acteurs de l'entreprise des «bonnes pratiques » auxquelles se référer. Mais ces travaux autour des indicateurs de performance peuvent également aller jusqu'à l'évaluation de performance.

Pour évaluer le fonctionnement d'une S.C, trois dimensions sont, à notre sens, essentielles à prendre en compte : le délai, la qualité et le coût (Hult et al., 2004). Ainsi que, la performance logistique est mesurée par la fiabilité des livraisons, la réduction des coûts, des délais, la conformité aux spécifications des clients, l'amélioration des processus et le time to market (autrement dit le délai de mise sur le marché de nouveaux produits).

\section{Conclusion}

D'où provient la performance logistique ? C'est la question à laquelle cet article tente de répondre. En effet, la performance est un concept complexe et multidimensionnel qui intègre différentes dimensions pour la définir et différents indicateurs de mesure car elle reste une affaire de perception et tous les acteurs n'ont pas la même perception de la performance. Elle est, par ailleurs, relative à la vision de l'entreprise, sa stratégie et ses objectifs. C'est dans ce sens que la performance d'une entreprise peut se mesurer sous différents angles et ne se limite pas uniquement à sa dimension financière. Ainsi pour l'évaluer, il est nécessaire d'effectuer des mesures au niveau de toutes ses dimensions et s'appuyer sur ses facteurs déterminants pour l'améliorer.

Par ailleurs, la logistique compte de nos jours parmi les leviers déterminants du devenir des organisations car sa performance influence significativement la performance globale des entreprises et leurs compétitivités. Pour s'en servir judicieusement pour cette fin, il est primordial de pouvoir la cerner dans ses différents aspects et s'en approprier notamment les sources génératrices de la performance logistique.

Pour conclure, après une large revue de la littérature sur la performance logistique et ses indicateurs de mesure, la fiabilité des livraisons, la réduction des coûts, des délais, la conformité aux spécifications des clients, l'amélioration des processus et le time to market sont les principaux éléments à prendre en considération pour arriver à une logistique performante, ils peuvent être regroupé en trois facteurs notamment le délai, la qualité et le coût.

\section{References:}

1. Armstrong, M. (2009). Handbook of Performance Management : An Evidence-based Guide to Delivering High Performance 4th edition, London, Kogan. 
2. Berrah, L. (2015). La quantification de la performance dans les entreprises manufacturières : de la déclaration des objectifs à la définition des systèmes d'indicateurs.

3. Berrah, L. (1997). Une approche d'évaluation de la performance industrielle, modèle d'indicateur et techniques floues pour un pilotage réactif.

4. Berrah, L. (2002). L'indicateur de performance, concepts et applications, Cépaduès, 2002, 170 pages.

5. Bhagwat, R., et Sharma, M. (2007). Performance measurement of supply chain management : A balanced scorecard approach. Computers \& Industrial Engineering. 53. 43-62. 10.1016/j.cie.2007.04.001.

6. Bouquin, H. (2004). Le contrôle de gestion, 2ème édition, Collection Que sais-je, Presses Universitaire de France, Paris.

7. Chantal Martin-Bonnefous, Alain Courtois. (2001). Indicateurs de performance 282 pages, parution le 15/07/2001.

8. Chen, Injazz \& Paulraj, Antony. (2004). Towards a Theory of Supply Chain Management. Journal of Operations Management. 22. 119-150. 10.1016/j.jom.2003.12.007.

9. Chow, G., Heaver, T. D. \& Henriksson, L. E. (1994). Logistics Performance : Definition and Measurement. International Journal of Physical Distribution \& Logistics Management, 24(1), p. 17-28.

10. Estampe, D. (2015). Performance de la Supply Chain et modèles d'évaluation. Editeur : ISTE. ISBN : 9781784060220.

11. Estampe, D., Lamouri, S., Paris, J., et Brahim-Djelloul, S. (2013). A framework for analysing supply chain performance evaluation models. International Journal of Production Economics - INT J PROD ECON. 142. 247-258. 10.1016/j.ijpe.2010.11.024.

12. Estampe, Dominique \& Lamouri, Samir \& Paris, Jean-Luc \& BrahimDjelloul, Sakina. (2013). A framework for analysing supply chain performance evaluation models. International Journal of Production Economics - INT J PROD ECON. 142. 247-258. 10.1016/j.ijpe.2010.11.024.

13. Fassio, Georges. (2006). Risques et performance d'un réseau industriel d'approvisionnement, essai d'identification et d'analyse des secteurs automobile, aéronautique et construction navale. Logistique \& Management. 14. 10.1080/12507970.2006.11516851.

14. Fender, M., et Pimor, Y. (2016). Logistique supply chain. ISBN :9782-10-074941-6

15. Forslund, H. (2012). Performance management in supply chains: Logistics service providers' perspective. International Journal of 
Physical Distribution \& Logistics Management. 42. 10.1108/09600031211225972.

16. Giraud F., Saulpic O., Naulleau G., Delmond M-H. et Bescos P-L. (2002), Contrôle de gestion et pilotage de la performance, Gualino éditeur.

17. Gunasekaran, A., Patel, C., et Mcgaughey, R. (2004). A Framework for Supply Chain Performance Measurement. International Journal of Production Economics. 87. 333-347. 10.1016/j.ijpe.2003.08.003.

18. Hult, G. Tomas M., Hurley, Robert., et Knight, Gary. (2004). Innovativeness: Its Antecedents and Impact on Business Performance. Industrial Marketing Management. 33. 429-438. 10.1016/j.indmarman.2003.08.015.

19. Hult, G. Tomas M., Hurley, Robert., et Knight, Gary. (2004). Innovativeness: Its Antecedents and Impact on Business Performance. Industrial Marketing Management. 33. 429-438. 10.1016/j.indmarman.2003.08.015.

20. Issor, Z. (2017). «La performance de l'entreprise : un concept complexe aux multiples dimensions ». Projectics / Proyéctica / Projectique, 2(2), 93-103. https://doi.org/10.3917/proj.017.0093

21. J.F. Le Clainche. (2001). Balancing scorecard et autres méthodes pour évaluer le score d'une entreprise, dans Indicateurs de performance.

22. Lorino, P. (2003). Méthodes et pratiques de la performance : le pilotage par les processus et les compétences. Paris, Editions d'Organisation.

23. Lorino, Paul. 1997. Méthodes et pratiques de la performance. Les Editions d'organisation, Paris, $512 \mathrm{p}$.

24. Monette, S., Bigras, M., et Guay, M.-C. (2011). The role of executive functions in school achievement at the end of Grade 1. Journal of $\begin{array}{llll}\text { experimental child psychology. } 109 . & 158-73 .\end{array}$ 10.1016/j.jecp.2011.01.008.

25. Neely, A., Gregory, M., et Platts, K. (2005). Performance measurement system design : A literature review and research agenda. International Journal of Operations \& Production Management. 25. 1228-1263. 10.1108/01443570510633639.

26. Paché, G., et Sauvage, T. (1999), La logistique : enjeux stratégiques, Vuibert Entreprise, Paris, 2e éd.

27. Panayides, P., et Lun, Y.H. (2009). The Impact of Trust on Innovativeness and Supply Chain Performance. International Journal of Production Economics. 122. 35-46. 10.1016/j.ijpe.2008.12.025.

28. Pichot, L. (2016). Stratégie de déploiement d'outils de pilotage de chaînes logistiques : apport de la classification. 
29. Richey, R., Roath, A., Whipple, J., et Fawcett, S. (2010). Governing Supply Chain Integration: Facilitators and Barriers. Journal of Business Logistics. 31. 237-256.

30. Saulquin J.Y. \& maupetit C. (2002), EVA, performance et évaluation bancaire.

31. Saulquin J.-Y. \& Schier G. (2007), « Des perceptions managériales aux pratiques RSE : une étude exploratoire », Gestion 2000, Vol. 24, n 6 , p. 181-195.

32. Wissler, M., Asquin, A., Marion, A., Christophe, E., et Vinot, D. (2012). Diagnostic de la performance de l'entreprise. Concepts et méthodes.

33. Wegelius-Lehtonen, Tutu. (2001). Performance measurement in construction logistics. International Journal of Production Economics. 69. 107-116. 10.1016/S0925-5273(00)00034-7.

34. Botta-Genoulaz, V., \& Khader, S., \& Rekik, Y., \& Campagne, J-P. (2011). Impact de la technologie RFID sur le management des chaines logistiques : une revue de littérature. 